\title{
BIOTECHNOLOGY AND ITS RELEVANCE TO SUGARCANE RESEARCH
}

\section{N. DHARMAWARDENE}

, Sugarcane Research Institute, 5/3 Udyana Road, Jayanthipura, Battaramulla.

Biotechnology may be regarded as the innovative application of biology for the production of commercially valuable materials which cannot be easily produced by conventional methods hitherto used in biology. So biotechnology is not a discipline by itself. It is an assortment of many scientific disciplines with a heavy leaning on biology, biochemistry, molecular biology, microbiology, chemical engineering etc. Therefore it is not quite correct to think of training youngsters at universities in biotechnology, rather it is more appropriate to think in terms of developing the major disciplines mentioned above. Our university courses should be strengthened in those disciplines which could ultimately lead to the synthesis which is biotechnology. This is what we are lacking. One cannot suddenly develop technologies out of a hat. The universities in Sri Lanka mainly serve a teaching function and have very little research activity. In such a situation little new knowledge is developed and the interaction between many basic disciplines in life sciences at a very high level hardly ever take place. Where there is no opportunity for life scientists to interact, no pragmatic applications of it could be conceived. This is the situation we are faced with today. Unless some meaningful action is taken to resolve this situation, biotechnology will not develop in Sri Lanka. There is very little original research or its applications in this field currently going on in Sri Lanka. We need to identify the limiting factors. Basic sciences and their applications cannot take root in this country this way. Although it is fashionable to talk of biotechnology as a single discipline in science, in reality it is not a single discipline. It is made up of a large number of basic scientific disciplines. Thus it requires the interaction of a number of different areas of expertise in life sciences, chemical and physical sciences. If we classify the presentations of this morning, they fall into basic disciplines such as physiology, biochemistry, microbiology etc. Thus this whole morning we have been talking about the use of such disciplines. Thus biotechnology is concerned with the use of basic sciences for an applied goal. This is the so called biotechnology.

Let us now consider basic scientific research and its application to solve the problems of the sugar industry. Our main theme of research is sugarcane variety improvement (Fig. 1). That is in essence creating as many genetic variants as possible which could give us a large pool of useful progenies to put through our selection programme. This is in one sense conventional biotechnology. However my definition earlier purposely excluded them. Here only the nonconventional technology which we use to obtain variants will be described. We use tissue culture techniques on sugarcane leaf explants from proven varieties, which may nevertheless be defective in one or two desirable characters, to obtain callus generated clones. Caliclones obtained by such methods are pooled with conventional progenies but these are rapidly advanced from the first ground nursery to the disease test stage unlike the others. Those proven at this stage are evaluated against the mother clone with respect 
to the defective characters. By this method we have now come up with two superior caliclones from the prominent variety grown here Co775. The new caliclones are superior in disease resistance and some other agronomic characters. These are now in the semi-commercial stage in the selection programme and soon they will be available to the local sugar industry. This is just one example of the use of basic research and its application to solve a given problem.

Now let me get on to the next line of activity which is showing good promise, namely the use of micro-organisms to control insect pests. About a year ago our microbiologists isolated two fungi which were found to attack and kill the sugar cane leaf hopper Pyrilla perpusilla. Pyrilla leaf hopper is a very serious pest of sugar cane here. Biological control is the only plausible answer. The two promising entomogenous fungi isolated by us namely Metarhizium anisopliae and Paecilomyces lilacinus have been successfully cultured in the laboratory on a large scale. They were then obtained in a form that could be sprayed using ordinary field sprayers. Field tests have shown that the latter fungus is more virulent than the former and this can bring down the adult leaf hopper population rapidly to levels which are no longer economically injurious. This is effective during times of the year when relative humidity is high.

In addition to the above we have been successful at isolating thermophilic, alcohol tolerant yeast strains which have a higher efficiency in converting molasses based sugar to alcohol. These new isolates SRISLC 102 and 111 have already proven their superiority at the Hingurana and Sevenegala distilleries. Once fully commercialised they will bring much benefit to the industry and country at large.

Lastly I wish to add that SRI is currently testing various blends of sugarcane based alcohol and petrol for use in internal combustion engines. We have successfully tested a mixture of petrol: alcohol in the ratio $75: 25$ in motor cycles and lawn mowers. This work is currently being pursued in collaboration with the Technical College, Ratmalana.

It is the sugar industry which has a cheap organised source of carbon for growing micro-organisms. Sugar cane molasses, a product of sugar manufacture, can be an ideal source for biotechnological production, and awaits exploitation for the country's benefit. This is the best challenge I can offer you this afternoon. 


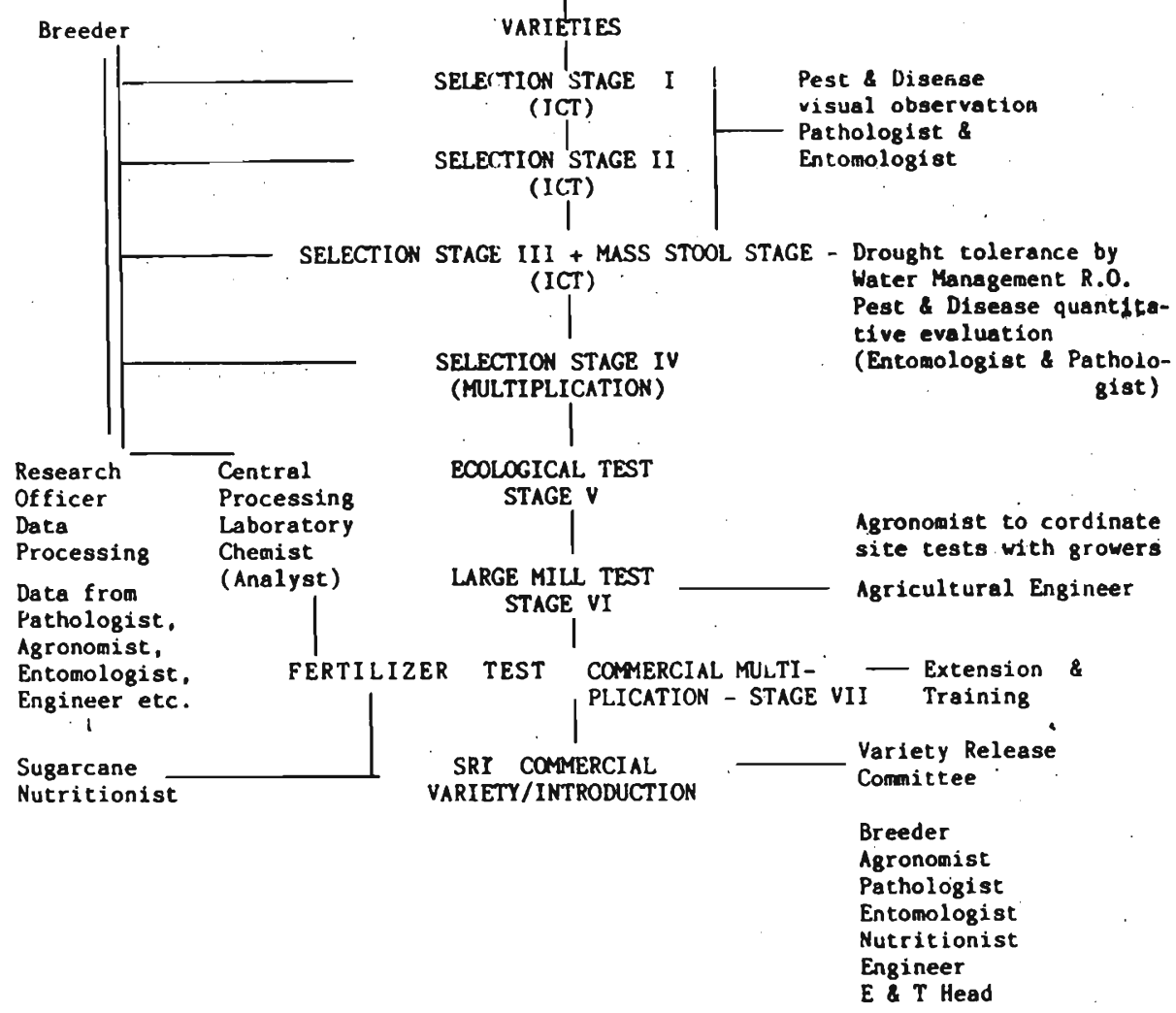

Figure 1: Scheme for sugarcane variety improvement 